\title{
A Season-of-Birth/DRD4 Interaction Predicts Maximal Body Mass Index in Women with Bulimia Nervosa
}

\author{
Robert D Levitan*,', Allan S Kaplan ${ }^{1,2}$, Caroline Davis ${ }^{3}$, Raymond W Lam ${ }^{4}$ and James L Kennedy ${ }^{1,5}$ \\ 'Mood and Anxiety Division, Department of Psychiatry, Centre for Addiction and Mental Health, University of Toronto, Toronto, ON, Canada; \\ ${ }^{2}$ Eating Disorders Program, Toronto General Hospital, University Health Network, University of Toronto, Toronto, ON, Canada; ${ }^{3}$ Department of \\ Psychology; York University, Toronto, ON, Canada; ${ }^{4}$ Department of Psychiatry, University of British Columbia, Toronto, ON, Canada; \\ ${ }^{5}$ Neurogenetics Section, Department of Psychiatry, Centre for Addiction and Mental Health, University of Toronto, Toronto, ON, Canada
}

\begin{abstract}
We have earlier reported that season of birth interacts with the hypofunctional 7-repeat (7R) allele of the dopamine-4 receptor gene (DRD4) to promote weight gain and obesity in women with seasonal affective disorder (SAD). This study examined whether this gene-environment interaction influences body weight regulation in women with bulimia nervosa (BN). In 188 female probands with BN, we performed an analysis of covariance predicting maximum lifetime body mass index (BMI) using season-of-birth, DRD4 genotype (7R present/absent), and past history of anorexia nervosa (yes/no) as independent variables, and age at maximum weight as the co-variate. Consistent with our SAD study, the birth-season $\times$ DRD4 interaction was a significant predictor of maximal BMI. Although in $\mathrm{SAD}$, the spring-birth/7R + group had markedly elevated maximal BMls and high rates of obesity, in this BN sample, the fall-birth/7R + group exhibited the highest BMI values ( $N=17$ : mean maximal $\mathrm{BMI}=28.2 \mathrm{~kg} / \mathrm{m}^{2}$ (SE 0.9) vs $25.2 \mathrm{~kg} / \mathrm{m}^{2}$ (SE 0.3 ) for all other probands combined $(N=17 \mathrm{I}) ; p=0.002)$. The lifetime rate of obesity $(\mathrm{BMI}>30)$ was also higher in the fall-birth/7R + vs 'other' group ( $29.9 \mathrm{vs}$ $8.8 \%$, respectively, $p=0.008$ ). These data offer further evidence that season of birth interacts with the $7 R$ allele of DRD4 to influence body weight regulation in female overeating populations.

Neuropsychopharmacology (2010) 35, 1729-1733; doi:10.1038/npp.2010.38; published online 24 March 2010
\end{abstract}

Keywords: bulimia nervosa; dopamine-4 receptor gene; birth season; obesity; thrifty phenotype hypothesis; developmental plasticity

\section{INTRODUCTION}

Developmental plasticity refers to the process by which genetic programs are modified in response to environmental conditions to shape physiology and behavior. In human beings, much of the work in this area has focused on the early origins of obesity and metabolic disorders, and is based on the fundamental notion that over the course of evolution, developing organisms would greatly enhance their chance of survival by matching long-term energy intake and metabolism to environmental conditions anticipated after birth. For example, the thrifty phenotype hypothesis of Hales and Barker (2001) proposes that a low-birth weight and long-term insulin resistance are adaptive if food supplies are scarce and likely to remain so over time. A thrifty phenotype is thought to be triggered by one or more signals of maternal malnutrition passing across the placenta to the developing fetus, thus providing

\footnotetext{
*Correspondence: Dr RD Levitan, Department of Psychiatry, University of Toronto, c/o CAMH, 250 College Street, room II26, Toronto, ON, Canada M5T IR8, Tel: + I 416535850 I ext. 4020, Fax: + I 416979 6821, E-mail: robert_levitan@camh.net Received 21 October 2009; revised II February 2010; accepted 23 February 2010
}

the fetus with a forecast of a sparse nutritional environment. If the prediction proves wrong and food supplies become abundant, the thrifty phenotype becomes a risk factor for obesity, diabetes, and cardiovascular disease.

We have recently proposed a seasonal thrifty hypothesis to explain the early developmental origins of obesity in the context of winter seasonal affective disorder (SAD; Levitan et al, 2006), a recurrent form of mood disorder characterized by overeating and weight gain in a majority of cases (Rosenthal et al, 1984). According to our model, one phenotype of SAD reflects the vestigial expression of a genetic program designed to preserve body mass in the face of predictable seasonal famines. The proposed trigger is an early signal of latitude transmitted in utero through maternal melatonin and/or diet (Levitan et al, 2006). As is the case with the original thrifty phenotype, such a process becomes maladaptive in a plentiful food environment, leading to high rates of obesity in SAD patients with this vulnerability.

The specific finding that inspired our thrifty model was an interaction between season-of-birth and the 7-repeat (7R) allele of the dopamine-4 receptor gene (DRD4) in predicting obesity in women with SAD (Levitan et al, 2006). DRD4 is of particular interest as a putative 'thrifty gene' for several reasons: (1) the 7R variant of DRD4 is hypofunctional 
(Asghari et al, 1995), and low-dopamine activity is thought to have a major function in overeating and obesity (Wang et al, 2001; Volkow and Wise, 2005; Stice et al, 2008); (2) there is mounting evidence that $7 \mathrm{R}$ carriers may have a unique ability to adapt appetitive behavior and body composition to anticipated climactic conditions (Levitan et al, 2006; Eisenberg et al, 2008); (3) there has been strong positive selection of the $7 \mathrm{R}$ allele in the last 40000 years of human evolution (Ding et al, 2002). It has been suggested that the ability of $7 \mathrm{R}$ to optimize weight and energy regulation under extreme environmental conditions, particularly in migrating populations, might have had a function in this regard (Levitan et al, 2004, 2006; Eisenberg et al, 2008).

This study looks at whether season of birth interacts with the 7R allele of DRD4 to predict weight gain and obesity in a second group of female overeaters, that is women with bulimia nervosa (BN). It was hypothesized that in a heterogenous group of women with $\mathrm{BN}$, the interaction of DRD4 genotype (defined by the presence or absence of the hypofunctional 7R allele) and birth season (spring/summer/ fall/winter) would be associated with increased maximal lifetime body mass indices (BMIs), thus replicating our overall findings in women with SAD.

\section{MATERIALS AND METHODS}

\section{Sample}

The current sample consisted of 188 consecutive female probands, 16-50 years of age, with DSM IV-defined BN with or without a past history of anorexia nervosa (AN). Ancestral information was available for 182 probands. Of these, $165(90.7 \%)$ were Caucasian, $10(5.5 \%)$ Oriental, 4 (2.2\%) West Indian, 2 (1.1\%) Native Canadian, and $1(0.5 \%)$ African American. To limit the impact of morbid obesity because of rare genetic variants on the current results, individuals with a maximal lifetime BMI of $>50$ were excluded. To simplify the interpretation of results, BN probands meeting full DSM-IV criteria for SAD were also excluded. Subjects were recruited from a well-established eating disorder treatment program or from the community through newspaper advertisements. They were initially screened on the telephone and then personally interviewed using the structured clinical interview for DSM IV (First et al, 2002) and the eating disorder examination (Cooper et al, 1989). Maximum and minimum weight achieved from age 16 years onward (excluding pregnancy) with corresponding heights and ages were obtained through self-report. BMI was calculated for each subject based on the formula (weight in kilograms/corresponding height in meters ${ }^{2}$ ).

Each subject was given an oral and written summary of the purposes, procedures, and potential risks of the project and gave informed written consent. The protocol was approved by the University of Toronto and University of British Columbia research ethics committees.

\section{Laboratory Methods}

Blood samples were sent to the Centre for Addiction and Mental Health neurogenetics laboratory. Genomic DNA was extracted from white blood cells using the high-salt method.
All genotyping of the DNA was performed blind to psychiatric diagnosis and vice versa. This was facilitated through a standard patient identification coding system used by our laboratory. The 48 base pair VNTR region in the third exon of DRD4 was amplified using polymerase chain reaction (PCR) techniques with primers and conditions earlier published (Lichter et al, 1993). The PCR products were visualized through gel electrophoresis performed in 3.5\% agarose prepared with ethidium bromide and $1 \mathrm{X}$ TBE (Tris, boric acid, EDTA).

\section{Statistical Methods}

The same statistical approach as described in our earlier manuscript was used (Levitan et al, 2006), except that a dichotomous categorical variable (history of AN vs no history of AN) was added as a factor to the current analysis. In summary, to study the main effect of genotype, two genotypic groups were defined based on whether a given proband did or did not carry at least one version of the hypofunctional 7R allele of DRD4 (designated the 7R and no7R groups, respectively). To study the main effect of birth season, four season-of-birth groups were defined based on the calendar year, that is spring births had occurred between 22 March and 21 June inclusive, summer births were between 22 June and 21 September inclusive, fall births between 22 September and 21 December inclusive, and winter births between 22 December and 21 March inclusive. The genotype $\times$ birth-season interaction thus included eight groups, including four with the $7 \mathrm{R}$ allele (7R/spring, 7R/summer, 7R/fall, and 7R/winter) and four without this allele (no7R/spring, no7R/summer, no7R/fall, and no7R/winter).

A 2 [genotype] $\times 4$ [season of birth] $\times 2$ [history of AN] analysis of covariance (ANCOVA) was carried out with maximum lifetime BMI as the dependent variable and age at maximal BMI as the co-variate. Where applicable, post hoc analyses used the least significant difference test.

The significance level for the main ANCOVA was set at $p<0.01$. Given the large number of study groups, which limits statistical power, a significance level of 0.05 was set for the main and interaction effects and post hoc tests, as was performed in our prior analyses of SAD probands.

\section{RESULTS}

\section{Sample Characteristics}

Error terms for unadjusted means are SDs and BMI measures are in $\mathrm{kg} / \mathrm{m}^{2}$. The mean current age of the sample was $25.2( \pm 6.2)$ years. The mean current BMI was 22.4 $( \pm 4.1)$, the mean minimal lifetime BMI was $18.6( \pm 2.6)$, and the mean maximum BMI was $25.5( \pm 4.3)$. Eighty probands $(42.6 \%)$ had a past history of AN.

The frequency of the common $4 \mathrm{R}$ and $7 \mathrm{R}$ alleles was 69.1 and $17.3 \%$, respectively, highly consistent with our prior study in women with SAD (Levitan et al, 2006). There was no deviation from Hardy-Weinberg equilibrium. There were 61 probands $(32.4 \%)$ with at least one version of the 7R allele. 
Table I General Linear Model Predicting Maximum Lifetime Body Mass Index (BMI) with DRD4 Genotype, Birth Season, and Past History of Anorexia Nervosa (AN), Co-varied by Age at Maximal $\mathrm{BMI}$ in 188 Women with Bulimia Nervosa

\begin{tabular}{|c|c|c|c|c|c|}
\hline & df & $\mathbf{F}$ & $p$-value & Partial $\eta^{2}$ & Power \\
\hline Overall model & 16 & 5.56 & $<0.000 \mid$ & 0.342 & 1.00 \\
\hline \multicolumn{6}{|l|}{ Source } \\
\hline Age at maximal BMI & । & 46.44 & $<0.0001$ & 0.214 & 1.00 \\
\hline DRD4 genotype ${ }^{a}$ & । & 7.39 & 0.007 & 0.041 & 0.77 \\
\hline Birth season ${ }^{b}$ & 3 & 3.20 & 0.025 & 0.053 & 0.73 \\
\hline Past AN & I & 5.81 & 0.017 & 0.033 & 0.67 \\
\hline DRD4 $\times$ birth season & 3 & 2.92 & 0.036 & 0.049 & 0.69 \\
\hline DRD4 $\times$ past AN & । & 0.11 & 0.743 & 0.001 & 0.06 \\
\hline Birth season $\times$ past $A N$ & 3 & 2.08 & 0.105 & 0.035 & 0.53 \\
\hline $\begin{array}{l}\text { DRD } 4 \times \text { birth season } \\
\times \text { past AN }\end{array}$ & 3 & 1.94 & 0.125 & 0.033 & 0.50 \\
\hline Error & |7| & & & & \\
\hline Total & 188 & & & & \\
\hline
\end{tabular}

Abbreviation: df, degrees of freedom.

${ }^{a}$ Genotype defined by the presence or absence of the 7-repeat allele of DRD4. ${ }^{\mathrm{b}}$ Spring = 22 March-2I June; Summer $=22$ June-2I September; Fall $=22$

September-2I December; Winter $=22$ December-2l March.

\section{Maximal BMI, DRD4, Season of Birth, and Past AN}

The mean maximal BMIs for carriers and non-carriers of the $7 \mathrm{R}$ allele were $26.4 \pm 5.3$ and $25.0 \pm 3.6$, respectively. The mean maximal BMIs based on birth-season groupings were spring $26.0 \pm 5.8(N=44)$; summer $24.6 \pm 2.8(N=45)$; fall $26.6 \pm 5.2(N=47)$; and winter $24.8 \pm 2.9(N=52)$. The mean maximal BMIs in probands with and without a history of AN were $24.4 \pm 3.8$ and $26.3 \pm 4.4$, respectively.

The results of the general linear model predicting maximal BMI with genotype, birth season, and AN history, with age at maximal BMI as a co-variate are summarized in Table 1. As shown, the overall model was highly significant $(\mathrm{F}=5.56, \mathrm{df}=16,171 ; p<0.0001)$, accounting for $34.2 \%$ of the variance in maximal lifetime BMI in this sample. The genotype $\times$ birth-season interaction and the main effects of genotype, birth season, and AN history were all statistically significant. None of the other interaction terms met statistical significance. Post hoc testing of birth-season effects revealed that $\mathrm{BN}$ probands with a fall birth had a greater mean maximal BMI than did probands born in either summer or winter at $p<0.05$. No other pairwise group differences related to birth season were identified at this step.

\section{Post hoc Testing of the DRD4/Birth-Season Interaction}

Figure 1 summarizes the marginal means for maximal BMI in the eight subgroups defined by the interaction of DRD4 genotype (7R present or absent) and season of birth (spring, summer, fall, and winter). Post hoc testing revealed that all of the significant pairwise group differences were attributable to the high mean maximal BMI in $7 \mathrm{R}$ carriers born in

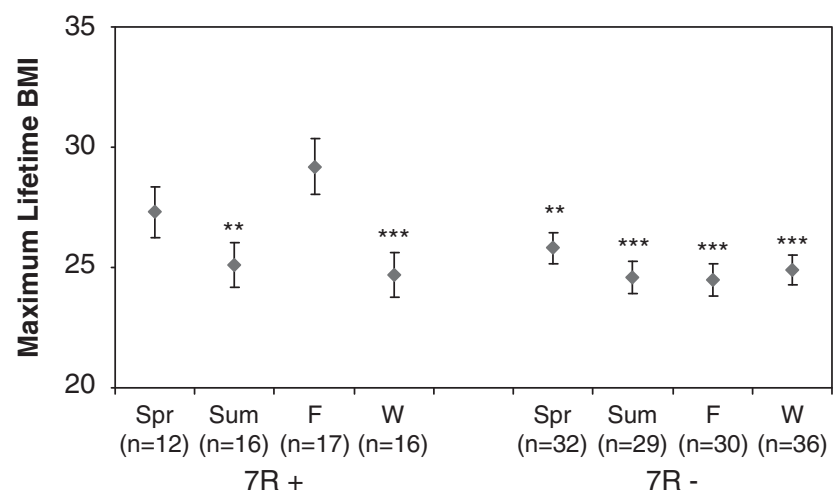

Figure I Marginal means for maximal lifetime BMI ( \pm SEM) in groups defined by the interaction of DRD4 genotype (7R present/absent) and season of birth in 188 women with Bulimia Nervosa. *** $<7 R+$ fall-birth group at $p \leqslant 0.01$; **** $<7 R+$ fall-birth group at $p \leqslant 0.001$.

the fall (ie the $7 \mathrm{R} /$ fall-birth group). As indicated, this group had a significantly higher value than four of the other interaction groups at $p \leqslant 0.001$, and two of the interaction groups at $p \leqslant 0.01$. The $7 \mathrm{R} /$ spring-birth group, which had by far the highest maximal BMI in our SAD sample (Levitan et al, 2006), did not differ significantly from any of the other interaction groups in this BN sample.

\section{Rates of Obesity}

On the basis of these findings, lifetime rates of obesity were next compared in the 7R/fall-birth and 'other' groups using Pearson $\chi^{2}$. The corresponding rates of obesity were $7 \mathrm{R} /$ fallbirth group $=5 / 17(29.4 \%) v s$ 'other' group $=15 / 171(8.8 \%)$; $\chi^{2}=6.93, \quad \mathrm{df}=1 ; \quad p=0.008 ; \quad$ ODDS ratio $=4.33, \quad 95 \%$ $\mathrm{CI}=1.34-13.96$.

\section{Analysis of Caucasian Probands}

To determine whether population stratification might have accounted for the current findings, we repeated our main analyses in the subgroup of probands of Caucasian ancestry $(N=165)$. In this sub-sample, the overall statistical model remained highly significant $(\mathrm{F}=4.89, \mathrm{df}=16,148, p<$ 0.001 ), accounting for $34.6 \%$ of the variance in maximal lifetime BMIs, whereas the genotype by birth-season interaction met a trend level of significance $(\mathrm{F}=2.16$, df $=3,148, p=0.095)$. Post hoc analysis revealed that the $7 \mathrm{R}-$ fall-birth group had a significantly higher mean maximal BMI than five of the other interaction groups at $p \leqslant 0.01$ and one of the interaction groups at $p<0.05$. The marginal means for maximal BMI in the $7 \mathrm{R} /$ fall-birth $(N=14)$ and 'other' $(N=151)$ groups defined above were $27.9 \mathrm{~kg} / \mathrm{m}^{2}$ (SE 1.0 ) and $25.2 \mathrm{~kg} / \mathrm{m}^{2}$ (SE 0.3$)$, respectively $(\mathrm{F}=6.64, \mathrm{df}=2$, $162, p=0.011)$. It would thus seem that limiting the analysis to Caucasian probands only did not have a major effect on the overall pattern of results. 


\section{DISCUSSION}

In showing that the 7R allele of DRD4 interacts with birth season to predict maximal BMI and obesity in women with $\mathrm{BN}$, the current results extend our prior findings in overeating women with SAD (Levitan et al, 2006). In this BN sample, 7R carriers born in the fall exhibited the highest maximal BMI values, whereas in our SAD sample, it was the 7R/spring-birth group who accounted for all of the significant effects. It would seem that during fetal and/or perinatal development, unknown factors tied to birth season interact with the hypofunctional $7 \mathrm{R}$ allele to influence maximal extremes of body weight in these female overeating populations. The overall pattern of results across our two studies suggests that different seasonal mechanisms might operate in these two disorders.

To explain why the season-of-birth effects found in BN were 6 months out-of-phase to our SAD data, more information on maternal eating disorders, food intake in pregnancy, birth weights, early feeding patterns, and the growth trajectories of our probands would be of great interest (Stewart et al, 1987; Treasure and Russell, 1988; Conti et al, 1998; Bulik et al, 1999, 2009; Sollid et al, 2004). Unfortunately, these variables were not included in the current protocol. Although important seasonal patterns of eating behavior, food preferences, and dieting exist in the population at large (Zahorska-Markiewicz, 1980; Zifferblatt et al, 1980; Attazardeh, 1983; Hardin et al, 1991; Yanovski et al, 2000), and in eating disorders per se (Lam et al, 1991; Brewerton et al, 1994; Levitan et al, 1994), no studies to date have examined these seasonal changes during pregnancy. It should be noted that both low and high extremes of birth weight can be associated with obesity over the lifespan (Hales and Barker, 2001; Phillips and Young, 2000). We speculate that in families with SAD, spring births may be associated with an increased risk of higher-birth weights because of fall/winter increases in total caloric intake in mothers during pregnancy. On the other hand, particularly in mothers with eating disorder pathology, fall births may be associated with sub-optimal weight gain and lower-birth weights because of lower-caloric intake and increased physical activity in the warmer months. In either case, the long-term risk for weight gain and obesity in the offspring should be increased, particularly for carriers of the $7 \mathrm{R}$ allele. Future prospective studies starting in fetal development are needed to test this and other seasonal hypotheses directly.

\section{Plasticity Models of the DRD4 Gene}

It has recently been suggested that the 7R allele of DRD4 is an example of a 'plasticity gene' variant which magnifies environmental influences on phenotypic development (Belsky et al, 2009). The more specific notion that climactic conditions can interact with the $7 \mathrm{R}$ allele to influence weight regulation over time is also an area of emerging interest. For example, a recent study in Africa showed that $7 \mathrm{R}$ is associated with increased fat-free mass in Ariaal men who migrate over long distances, but not in their sedentary relatives (Eisenberg et al, 2008). Taken together with our findings in $\mathrm{SAD}$ and $\mathrm{BN}$, this suggests that the $7 \mathrm{R}$ allele may enable human beings to match nutritional intake and anthropomorphic makeup with anticipated climactic conditions relevant to food availability, energy storage, and energy usage. This adaptive function could help explain the rapid increase in the frequency of the $7 \mathrm{R}$ allele in recent human evolution (Ding et al, 2002). Although adaptive in strained environments and in migrating populations, this plasticity becomes a risk factor for obesity when circumstances change, leading to the widespread availability of highly palatable foods and the sedentary lifestyle typical of modern developed countries.

\section{Limitations and Future Directions}

The overall sample size for this study was small, particularly as it relates to $\mathrm{G} \times \mathrm{E}$ effects, and much larger studies are needed to replicate the current findings. Notwithstanding, this was a replication sample based on our earlier findings in SAD, and the overall pattern of results is highly consistent with our prior data. The specific finding of a 7R/fall-birth interaction predicting high BMIs and obesity was not predicted $a$ priori.

As a group, $\mathrm{BN}$ patients are more heterogenous than $\mathrm{SAD}$ patients, suggesting that many factors merit consideration when interpreting the overall results. On the basis of the high rate of past AN in probands with BN, we included past AN in the current analyses. Although the three-way interaction of genotype, season of birth, and AN history did not reach statistical significance, a general trend in this direction suggests that a three-way effect might emerge in future work. This is of potential relevance in that prior research has found season-of-birth effects in the AN population per se (Rezaul et al, 1996; Crisp et al, 2007). Another factor that was not part of the current protocol relates to the potential function of medications in shaping maximal BMIs. For example, many BN probands would have been on one or more anti-depressants over time, which can lead to weight gain and/or prevent weight gain in many cases. Lifetime medication histories will thus be an important consideration in future work of this type.

If replicated in larger samples, the current results might have implications for the prevention of obesity in eatingdisordered families. For example, in families with highdietary restraint and a high frequency of the $7 \mathrm{R}$ allele, seasonally based dietary management during pregnancy might prevent unusually low (or high) birth weights and at least some cases of obesity in the next generation. For fall births occurring at northern latitudes, limiting infants' exposure to cold temperatures in the first 6 months of life might also have a function in this regard (Phillips and Young, 2000). Pending future investigation of these $\mathrm{G} \times \mathrm{E}$ interactions in the population at large, broader application of such strategies might also prove useful in future years.

\section{ACKNOWLEDGEMENTS}

We thank Bronwyn Mackenzie and Subi Tharmalingam for their help with data collection and data management, and Tamara Arenovich for statistical advice. Project funding and personal funding for RDL were provided by the Ontario Mental Health Foundation. 


\section{DISCLOSURE}

The authors declare no conflict of interest.

\section{REFERENCES}

Asghari V, Sanyal S, Buchwaldt S, Paterson A, Jovanovic V, Van Tol HH (1995). Modulation of intracellular cyclic AMP levels by different human dopamine D4 receptor variants. J Neurochem 65: 1157-1165.

Attazardeh F (1983). Seasonal variation in stature and body weight. Int J Orthod 21: 3-12.

Belsky J, Jonassaint C, Pluess M, Stanton M, Brummett B, Williams R (2009). Vulnerability genes or plasticity genes? Mol Psychiatry 14: $746-754$.

Brewerton TD, Krahn DD, Hardin TA, Wehr TA, Rosenthal NE (1994). Findings from the Seasonal Pattern Assessment Questionnaire in patients with eating disorders and control subjects: effects of diagnosis and location. Psychiatry Res 52: 71-84.

Bulik CM, Sullivan PF, Fear JL, Pickering A, Dawn A, McCullin M (1999). Fertility and reproduction in women with anorexia nervosa: a controlled study. J Clin Psychiatry 60: 130-135.

Bulik CM, Von Holle A, Siega-Riz AM, Torgersen L, Lie KK, Hamer $\mathrm{RM}$ et al (2009). Birth outcomes in women with eating disorders in the Norwegian mother and child cohort study (MoBa). Int J Eat Disord 42: 9-18.

Conti J, Abraham S, Taylor A (1998). Eating behavior and pregnancy outcome. J Psychosom Res 44: 465-477.

Cooper Z, Cooper PJ, Fairburn CG (1989). The validity of the eating disorder examination and its subscales. $\mathrm{Br} J$ Psychiatry 154: $807-812$.

Crisp A, Gowers S, Joughin N, McChellan C, Rooney B, Nielsen S et al (2007). Anorexia and season of birth. Eur Eat Disord Rev 14: 144-146.

Ding YC, Chi HC, Grady DL, Morishima A, Kidd JR, Kidd KK et al (2002). Evidence of positive selection acting at the human dopamine receptor D4 gene locus. Proc Natl Acad Sci USA 99: 309-314.

Eisenberg DT, Campbell B, Gray PB, Sorenson MD (2008). Dopamine receptor genetic polymorphisms and body composition in undernourished pastoralists: an exploration of nutrition indices among nomadic and recently settled Ariaal men of northern Kenya. BMC Evol Biol 8: 173.

First MB, Spitzer RL, Williams JB, Gibbon M (2002). Structured Clinical Interview for DSM-IV-Patient Edition (SCID-P). American Psychiatric Press: Washington.

Hales CN, Barker DJ (2001). The thrifty phenotype hypothesis. Br Med Bull 60: 5-20.

Hardin TA, Wehr TA, Brewerton T, Kasper S, Berrettini W, Rabkin J et al (1991). Evaluation of seasonality in six clinical populations and two normal populations. J Psychiatr Res 25: 75-87.
Lam RW, Solyom L, Tompkins A (1991). Seasonal mood symptoms in bulimia nervosa and seasonal affective disorder. Compr Psychiatry 32: 552-558.

Levitan RD, Kaplan AS, Levitt AJ, Joffe RT (1994). Seasonal fluctuations in mood and eating behavior in bulimia nervosa. Int J Eat Disord 16: 295-299.

Levitan RD, Masellis M, Basile VS, Lam RW, Kaplan AS, Davis $C$ et al (2004). The dopamine-4 receptor gene associated with binge eating and weight gain in women with seasonal affective disorder: an evolutionary perspective. Biol Psychiatry 56: 665-669.

Levitan RD, Masellis M, Lam RW, Kaplan AS, Davis C, Tharmalingam $S$ et al (2006). A birth season/DRD4 gene interaction predicts weight gain and obesity in women with seasonal affective disorder: a seasonal thrifty phenotype hypothesis. Neuropsychopharmacology 11: 2498-2503.

Lichter JB, Barr CL, Kennedy JL, Van Tol HH, Kidd KK, Livak KJ (1993). A hypervariable segment in the human dopamine receptor D4 (DRD4) gene. Hum Mol Genet 2: 767-773.

Phillips DI, Young JB (2000). Birth weight, climate at birth and the risk of obesity in adult life. Int J Obes Relat Metab Disord 24: 281-287.

Rezaul I, Persaud R, Takei N, Treasure J (1996). Season of birth and eating disorders. Int J Eat Disord 19: 53-61.

Rosenthal NE, Sack DA, Gillin JC, Lewy AJ, Goodwin FK, Davenport $\mathrm{Y}$ et al (1984). Seasonal affective disorder. A description of the syndrome and preliminary findings with light therapy. Arch Gen Psychiatry 41: 72-80.

Sollid CP, Wisborg K, Hjort J, Secher NJ (2004). Eating disorder that was diagnosed before pregnancy and pregnancy outcome. Am J Obstet Gynecol 190: 206-210.

Stewart DE, Raskin J, Garfinkel PE, MacDonald OL, Robinson GE (1987). Anorexia nervosa, bulimia, and pregnancy. Am J Obstet Gynecol 157: 1194-1198.

Stice E, Spoor S, Bohon C, Small DM (2008). Relation between obesity and blunted striatal response to food is moderated by TaqIA A1 allele. Science 322: 449-452.

Treasure JL, Russell GF (1988). Intrauterine growth and neonatal weight gain in babies of women with anorexia nervosa. $\mathrm{Br} \mathrm{Med} \mathrm{J}$ (Clin Res Ed) 296: 1038 .

Volkow ND, Wise RA (2005). How can drug addiction help us understand obesity? Nat Neurosci 8: 555-560.

Wang GJ, Volkow ND, Logan J, Pappas NR, Wong CT, Zhu W et al (2001). Brain dopamine and obesity. Lancet 357: 354-357.

Yanovski JA, Yanovski SZ, Sovik KN, Nguyen TT, O'Neil PM, Sebring NG (2000). A prospective study of holiday weight gain. $N$ Engl J Med 342: 861-867.

Zahorska-Markiewicz B (1980). Weight reduction and seasonal variation. Int J Obes 4: 139-143.

Zifferblatt SM, Curtis CS, Pinsky JL (1980). Understanding food habits. J Am Diet Assoc 76: 9-14. 